\title{
Poster Abstract: Maximizing Accuracy of Fall Detection and Alert Systems Based on 3D Convolutional Neural Network
}

\author{
Seokhyun Hwang \\ Hanyang University \\ hsh2438@hanyang.ac.kr \\ Homin Park \\ Hanyang University \\ hominpark@hanyang.ac.kr
}

\begin{abstract}
We present a deep-learning-based approach to maximize the accuracy and reliability of vision-based fall detection and alert systems. The proposed approach utilizes a 3D convolutional neural network (3D-CNN) to analyze the continuous motion data obtained from depth cameras and exploits a data augmentation method to do away with overfitting. Our preliminary evaluation results demonstrate that it achieves the classification accuracy of up to $96.9 \%$.
\end{abstract}

\section{CCS CONCEPTS}

-Computing methodologies $\rightarrow$ Activity recognition and understanding; Neural networks;

\section{KEYWORDS}

IoT applications, fall detection, deep learning, 3D convolutional neural network, elderly care

ACM Reference format:

Seokhyun Hwang, DaeHan Ahn, Homin Park, and Taejoon Park. 2017. Poster Abstract: Maximizing Accuracy of Fall Detection and Alert Systems Based on 3D Convolutional Neural Network. In Proceedings of The 2nd ACM/IEEE International Conference on Internet-of-Things Design and Implementation, Pittsburgh, PA USA, April 2017 (IoTDI 2017), 2 pages.

DOI: $10.1145 / 3054977.3057314$

\section{INTRODUCTION}

With the advent of Internet of Things (IoT), smart homes are envisioned to provide various convenient services including health care. Among many health care services, fall detection and alert services have attained public attention since more than one out of four elderly people experiences fall accidents at least once a year. The fact that there are 46 million community-dwelling elderly in United States and about 30\% of them are living alone, aggravates the risks of falls. Since fall incidents may cause serious injuries

This work was supported in part by the National Research Foundation of Korea(NRF) grant funded by the Korea government (MSIP) (No. NRF-2016R1E1A2A01939769).

Permission to make digital or hard copies of all or part of this work for personal or classroom use is granted without fee provided that copies are not made or distributed for profit or commercial advantage and that copies bear this notice and the full citation on the first page. Copyrights for components of this work owned by others than ACM must be honored. Abstracting with credit is permitted. To copy otherwise, or republish, to post on servers or to redistribute to lists, requires prior specific permission and/or a fee. Request permissions from permissions@acm.org.

IoTDI 2017, Pittsburgh, PA USA

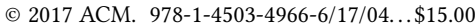

DOI: $10.1145 / 3054977.3057314$

\author{
DaeHan Ahn \\ Daegu Gyeongbuk Institute of Science and Technology \\ daehan@dgist.ac.kr \\ Taejoon Park \\ Hanyang University \\ taejoon@hanyang.ac.kr
}

preventing those living alone from calling 911 for helps, it is critically important to develop autonomous and reliable fall detection systems to accurately detect instances of falls in real time.

Most of elderly people suffer from poor muscle conditions, decreased strengths, and loss of flexibility. Hence, they are prone to falling down, possibly slowly, while bending, reaching, or rising from a furniture. But, to the best of our knowledge, previous works cannot adequately address this kind of slow falls, a serious threat to the elderly people. Clearly, it is important to consider all kinds of fall incidents including slow falls.

State-of-the-art camera-based fall detection solutions can be categorized into either inactivity analyses, 3D head motion analyses, or detection of body shape changes [3]. The inactivity-based solutions trigger an alarm when the resident is found to be motionless for a certain period of time, while those based on 3D head motion analyses rely on the fact that a vertical portion of motion is faster than the horizontal portion when falling down. The methods based on body shapes, on the other hand, analyze the body shape changes monitored throughout a fall (i.e., from standing to lying down), thus producing more accurate results that the former two solutions.

More specifically, Ma et al. [2] proposed a fall detection method that extracts curvature scale space features of human silhouettes and classifies them with an extreme learning machine to achieve up to $86.83 \%$ accuracy. Yoon et al. [4] presented a system using 3D skeletal joint data from Microsoft Kinect to analyze the fall behavior when the body shape of the resident has changed over a certain amount of time. However, the 3D skeletal joint data cannot accurately keep track of changes to the body shape when the resident is lying down. Most importantly, these solutions lack in the capability of detecting slow falls.

To counter the deficiencies of existing systems, we propose an approach to maximize the accuracy and reliable of vision-based fall detection and alert systems by exploiting deep learning. That is, we employ a 3D Convolutional Neural Network to directly analyze a video stream of continuous motions of a subject obtained when falling down. Accordingly, the proposed system is resilient against distortions of the skeletal structure as opposed to ones based on skeletal information. The proposed system is also capable of detecting slow falls once trained, because it captures subjects' behavior by using 3D-CNN. In general, a huge amount of training data significantly enhances the performance of 3D-CNN without overfitting (i.e., fitting much closely to the training data), but it is very costly to collect enough data from actual falls. To address this issue, we reinforce our proposed system with a data augmentation technique 


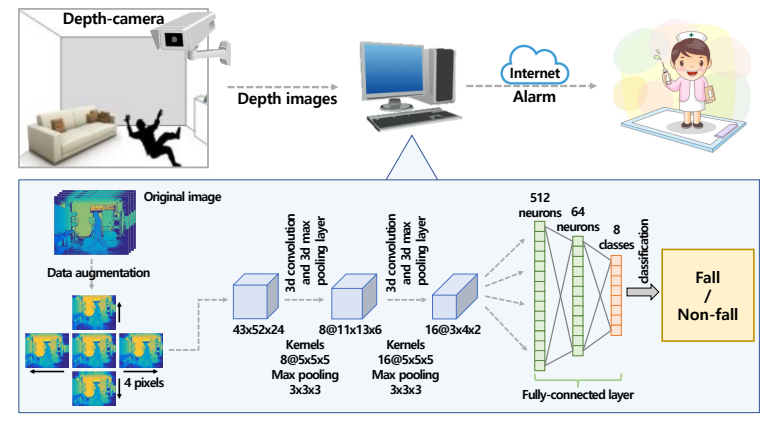

Figure 1: System Overview

that randomly generates training data in a virtual space. Our preliminary results demonstrate that the proposed system achieves the classification accuracy of up to $96.9 \%$. We plan to further evaluate our system to show it produces accurate results regardless of speed of falls, body orientations, and sensing distances.

\section{PROPOSED APPROACH}

As shown in Fig. 1, our proposed system includes the depth camera deployed in the house or nursing home captures depth video frames and sends them to a local computer. After receiving the depth video, the computer: 1 ) resizes (or down-samples) the input video to produce video frames with a dimension of $52 \times 43 \times 24$; 2) executes an inference/classification process by using a pre-trained 3D-CNNs model; and 3) if detecting a fall, generates an alarm by transmitting the classification results to the health care provider.

For system development and evaluation, we use a fall detection dataset v2 from the Telecommunication Systems Team (TST) as the input video [1]. The dataset contains 264 actions which is comprised of 8 categories, 4 activities of daily living (sitting, grasping, walking, and laying down), and 4 types of simulated falls (fall forward, fall backward, fall to the side, and fall backward ending up sitting) performed by 11 young actors. Depth frames, acceleration streams, skeleton joints are available but we utilize only the depth frames which have a resolution of $512 \times 424$ captured by the Microsoft Kinect v2.

The 3D-CNN in our proposed system consists of two convolution layers, two max-pooling layers and three fully-connected layers. We use a rectified linear unit (ReLU) activation function for maxpooling layers and a sigmoid activation function for fully-connected layers. Because the sigmoid activation may suffer from gradient vanishing caused by repeated multiplications of small values, it has a range between 0 and 1 . By contrast, the ReLU activation function is free of the gradient vanishing problem and even accelerates the convergence of gradient because it's linear. The fully-connected layer performs softmax computation for classification between fall and fall-like normal behavior.

As mentioned earlier, if we do not have an adequate amount of real data, the proposed 3D-CNN model would suffer from the overfitting problem. To avoid this problem, we apply data augmentation before training by using one of the existing methods, e.g., [? ], to significantly amplify the amount of data allowing spatial diversity. With data augmentation, depth videos are translated into 4 pixels along the 4 directions (left, right, up, down) to generate four virtual frames from each real frame.

To effectively train the 3D-CNN model, it is important to minimize a cost function by optimizing weights and biases. We use cross-entropy as a cost function to make training faster because the computational complexity of cross-entropy is much lower than ones based on mean squared errors. It performs optimization via a gradient descent optimizer with mini batches. We also apply a process of drop-out (with a ratio of 0.5 ) to the fully-connected layer. The drop-out randomly sets outputs as 0 during back propagation to reduce overfitting by preventing complex co-adaptations.

\section{PRELIMINARY RESULTS}

We conducted a total of 5 random trials. For each trial, we randomly extracted 240 and 24 videos for training and evaluation purposes, respectively, from the TST fall detection dataset.

We first evaluated the performance of our proposed system without applying data augmentation. As detailed in Table 1, the preliminary evaluation results reveal that it achieves $69.6 \sim 78.7 \%$ accuracy in 5 trials. We then applied data augmentation before 3D-CNN training to generate $5 \times$ larger training dataset and evaluated the system under the same conditions. The results of this evaluation show that its performance is significantly improved and achieves the classification accuracy of $92.4 \sim 96.9 \%$.

Table 1: Classification results of the proposed system in two configurations, i.e., with and without data augmentation.

\begin{tabular}{|c|c|c|c|c|c|}
\hline Trial & 1 & 2 & 3 & 4 & 5 \\
\hline 3D-CNN only & $71.2 \%$ & $77.2 \%$ & $72.7 \%$ & $\mathbf{7 8 . 7 \%}$ & $69.6 \%$ \\
\hline 3D-CNN+data augmentation & $93.9 \%$ & $\mathbf{9 6 . 9 \%}$ & $93.9 \%$ & $92.4 \%$ & $93.9 \%$ \\
\hline
\end{tabular}

\section{CONCLUSION}

We proposed a novel approach for constructing reliable fall detection and alert systems, which is based on the 3D-CNN model to directly process the continuous motion data from depth cameras. The proposed system overcame the overfitting issue by applying the data augmentation technique and achieved the classification accuracy of up to $96.9 \%$. As a future work, we plan to apply a background subtraction method as well as optimize/validate our system for detecting all kinds of falls including slow falls in a robust manner.

\section{REFERENCES}

[1] Samuele Gasparrini, Enea Cippitelli, Ennio Gambi, Susanna Spinsante, Jonas Whsln, Ibrahim Orhan, and Thomas Lindh. 2016. Proposal and experimental evaluation of fall detection solution based on wearable and depth data fusion. In In ICT Innovations 2015. Ohrid, Macedonia, 99-108.

[2] Xin Ma, Haibo Wang, Bingxia Xue, Mingang Zhou, Bing Ji, and Yibin Li. 2014. Depth-based human fall detection via shape features and improved extreme learning machine. IEEE journal of biomedical and health informatics 18, 6 (2014), 1915-1922.

[3] Yoosuf Nizam, Mohd Norzali Haji Mohd, and M. Mahadi Abdul Jamil. 2016. A Study on Human Fall Detection Systems: Daily Activity Classification and Sensing Techniques. International fournal of Integrated Engineering 8, 1 (January 2016), 35-43.

[4] Hee Jung Yoon, Ho-Kyeong Ra, Taejoon Park, Sam Chung, and Sang Hyuk Son. 2015. FADES: Behavioral detection of falls using body shapes from 3D joint data. fournal of Ambient Intelligence and Smart Environments 7, 6 (2015), 861-877. 